\title{
IMAGE CLASSIFICATIONS ON COVID 19 CXR IMAGES USING AUTO COLOR CORRELOGRAM FILTER
}

\author{
S. M. Manimegalai ${ }^{1}$, Dr.T.Ramaprabha ${ }^{2}$ \\ Research Scholar, PG and Research Department of Computer Science and Computer Application, \\ Vivekanandha College of Arts and Science for Women (A), Elayampalayam- 637205. Tamil Nadu, India. ${ }^{1}$ \\ Professor, Department of Information Technology, Nehru Arts and Science College, Coimbatore, Tamil Nadu, \\ India. $^{2}$ \\ megalaianand2@gmail.com¹, ramaradha1971@gmail.com²
}

\begin{abstract}
:
In this current situation necessary to control the spread of virus by using computer-based analysis for the purpose of fast detection and reliable of corona virus disease (COVID-19) to reduce the issues on medical field. In image processing techniques, Chest $\mathrm{X}$ ray imaging are having more benefits like very cheap, portability, fast and easily can handle. This paper explores the effect of various popular image enhancement techniques. This research work finds that the Random Forest classifier is producing highest accuracy value precision value, recall value, Mathews correlation coefficient value, ROC value, PRC value and Kappa value which are $80.96 \%$ of accuracy level, 0.81 of precision value, 0.81 of recall value, 0.72 of Mathews Correlation value, 0.95 of Receiver Operating Characteristic value, 0.87 of Precision Recall value and 0.70 of kappa statistic value. It is having lowest Mean Absolute Error (MAE) value, Root Mean Squared Error (RMSE) value, Relative Absolute Error (RAE) value, Root Relative Squared Error (RRSE) value which is accordingly 0.14 of MAE value, 0.20 of RMSE value, $43.73 \%$ of RAE value and $64.40 \%$ of RRSE value. Finally this research work recommends that the Random Forest classifier is most recommend model by using Auto Color Correlogram Filter in image classifications.
\end{abstract}

Keywords: Auto Color Correlogram Filter, BayesNet, Sequential Minimal Optimization, lazy classifier and Tree classifier.

\section{Introduction}

Image enhancement process has group of methods implemented to develop the visual form of an image for instance eliminating blur and noise of the image, which in try upgrade contrast and also provides more information of an image. contrast.

The snapshots are taken through smart phones and some other electronic device are generally of poor

So that, this type of images essentials boost algorithms to advance its contrast. It is compulsory to advance the overall excellence of the image, which recovers the spatial features of those images.

The important persistence of image augmentation is to improve the interpretability or else observation of facts contained in the image for human observers or feature extraction an image that is individually improved than the novel image by altering the pixel's strength of the input image. A vital concern is not to change the details throughout the image improvement process.

The rest of this paper is organized as follows: Section 2 represents that the related works; section 3 shows that the materials and methods; Section 4 presents that the results and discussions; finally conclusion presents in section 5 .

\section{Related Works}

In Worldwide health care system is facing the one of the vital issues is Coronavirus Disease 2019 (COVID 19)[1,2,3-13]. Many effective approaches have been proposed to diagnose COVID-19 using chest radiography images including X-rays and CT scans. Now, there are 235,308,300 affected by corana cases and 4,809,284 death cases in the world, as of October 32021 [14]. Coronavirus Disease 2019 analysis like low accuracy, precision and recall and related statistics measuremnets is conceded out by Reverse Transcription Polymerase Chain Reaction (RT-PCR [15-18]. Primary analysis of a virus growths the probabilities for fruitful treatment of diseased patients and also decreases the hazards of dispersal in the communal for a transmissible disease like COVID-19. Chest Xray (CXR) or Computed tomography (CT) are usual technique for identifying and analysis the lung related 
diseases like pneumonia [19], Tuberculosis [20] and also it is convenient in COVID-19 detection also [21, 22]. One of the assistances of CXR is the capability to achieve them simply consuming portable X-ray machines provided that faster, accurate COVID- 19 analysis [21, 22-23]. CXRs are established to be possible for distinguishing COVID-19 with the help of artificial intelligence in machine learning techniques and are also less injurious for human body associated to CT. [22-23]. There is a demand for medical image improvement to assistance clinicians make accurate with good analysis of illness [15, 17, 21-23].

\section{Materials and Methods}

In this section focuses on the Materials and methods on this research work. Covid 19 dataset borrowed from Kaggle.

Table 1: Meta data of Dataset

\begin{tabular}{|c|c|c|c|c|c|}
\hline S.No & $\begin{array}{l}\text { Data Collected and } \\
\text { Created from Team of } \\
\text { Researches (Country) }\end{array}$ & Category & $\begin{array}{l}\text { Image } \\
\text { Size }\end{array}$ & $\begin{array}{l}\text { Number of } \\
\text { Images }\end{array}$ & $\begin{array}{c}\text { Sample Size } \\
(\text { Random }-\mathbf{1 0 \%})\end{array}$ \\
\hline 1 & \multirow{4}{*}{$\begin{array}{l}\text { a) Qatar } \\
\text { University, } \\
\text { [Doha,Qatar], } \\
\text { b) University of } \\
\text { Dhaka, } \\
\text { c) Bangladesh, } \\
\text { d) Pakistan and } \\
\text { e) Malaysia } \\
\end{array}$} & Covid19 & $256 \times 256$ & 3616 & 362 \\
\hline 2 & & Normal & $256 \times 256$ & 10192 & 1019 \\
\hline 3 & & $\begin{array}{l}\text { Lung } \\
\text { Opacity }\end{array}$ & $256 \times 256$ & 6012 & 601 \\
\hline 4 & & $\begin{array}{l}\text { Viral } \\
\text { Pneumonia }\end{array}$ & $256 \times 256$ & 1345 & 135 \\
\hline & & & Total & 21170 & 2117 \\
\hline
\end{tabular}

\section{Methods:}

The following methods are implemented in this research work.[24-27]

1) Borrowed dataset

2) Data preprocessing

3) Apply Auto Color Correlagram Filter

4) Apply for machine learning algorithms
a) BayesNet of Bays category
b) SMO of Function category
c) IBK of Lazy category
d) Bagging of Ensemble Category
e) JRip of Rules category
f) Random Forest of Trees category

5) To get Optimal results

6) Find a best Model

These above methods implemented in one of the leading and open source tools which is Weka 3.9.5 for obtain an efficient result. In this research work takes only $10 \%$ of overall dataset with 10 fold cross Validation for all classifications. 
Dataset Collection( Covid 19 Image Dataset)

Data Preprocessing

Feature Extraction (Auto Color Correlogram Filter)

Classification with 10 fold cross validation

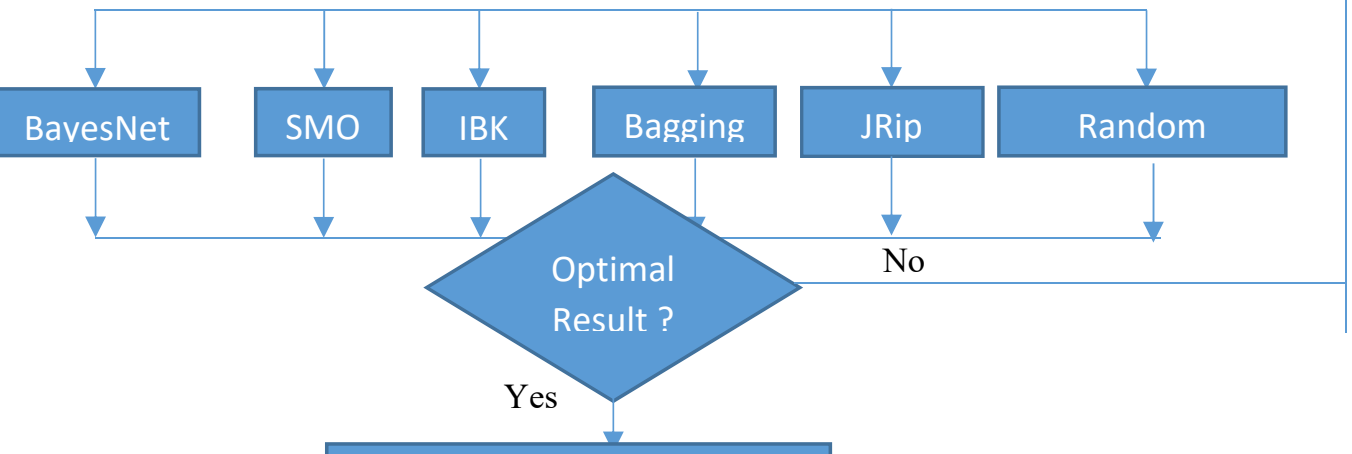

Model Evaluation

Figure 1: Proposed System Architecture

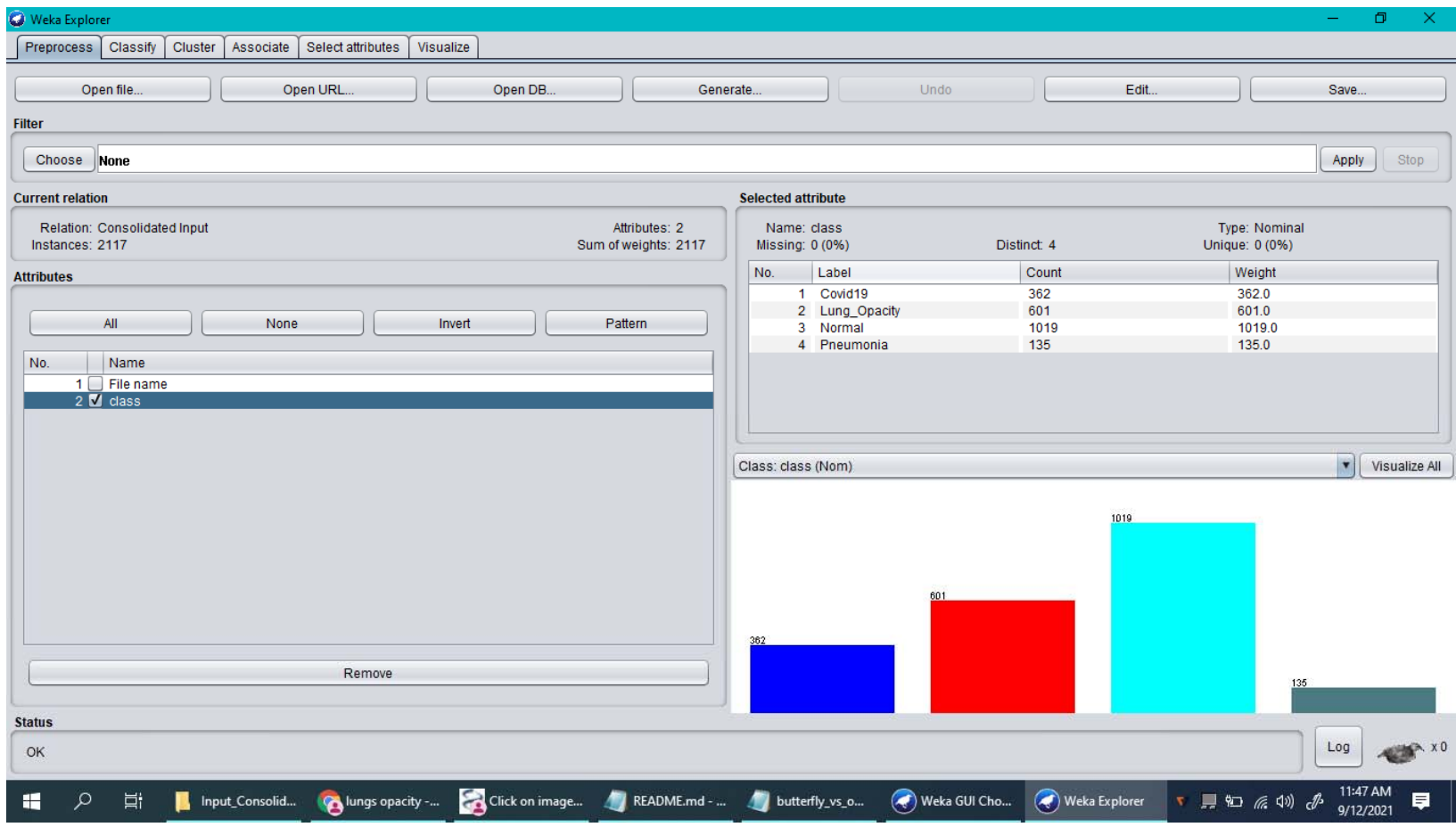

Figure 2: Overview of multiclass in Weka3.9.5 


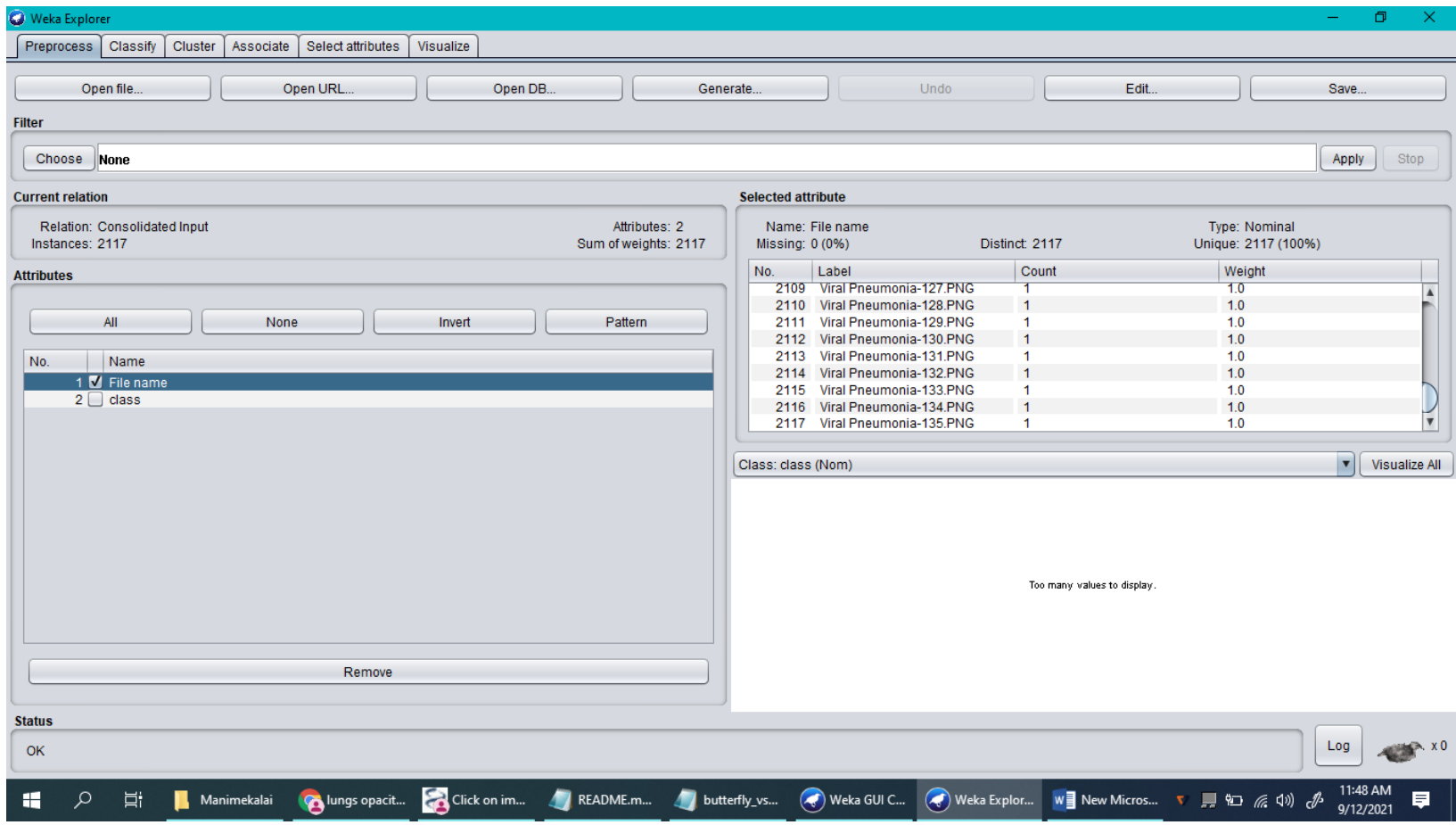

Figure 3: Representation of Filename in Dataset

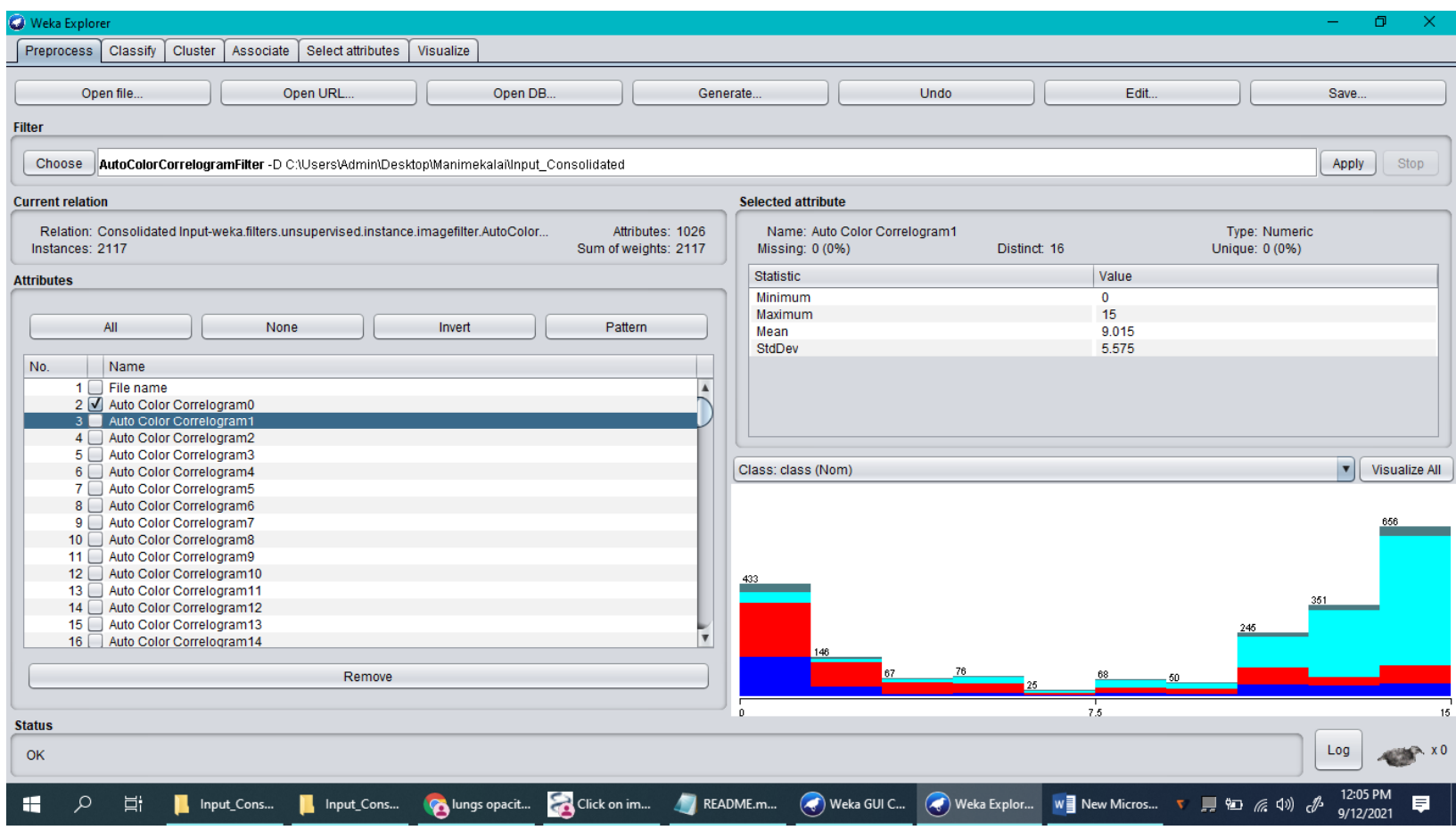

Figure 4. Implementation of Auto Color Correlagrom filter for classification 


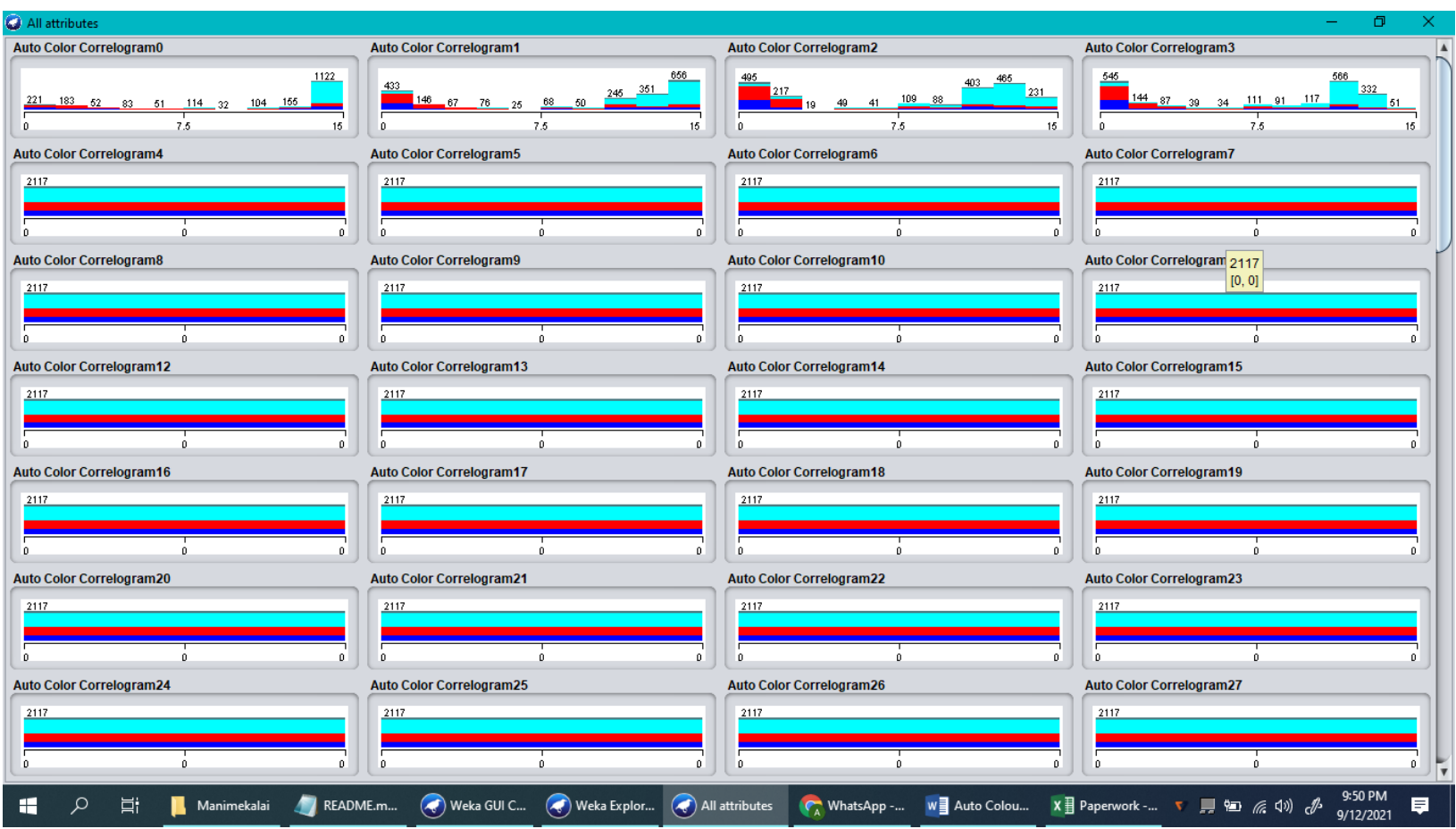

Figure 5: Visualization of file names applied with Auto Color Correlogram Filter

\section{Results and Discussions}

In this section discusses results and analysis of this research work. This experiment is realized by applying several classifiers. Namely, BayesNet classifier of Bays category, SMO model (Support Vector Machine) of function category, Instance Based Classifier of Lazy category, Bagging classifier of ensemble category, JRip classifier of Rules category and Random Forest classifier of Trees category for bringing out the optimal results. Such as accuracy, Kappa static values, Mean Absolute Error, Root Mean Squared Error value, Relative Mean Squared Error value, Root Relative Squared Error value, Precision value, Recall value, F-Measure value, Mathews Correlation Coefficient value, Receiver Operating Characteristic curve, Precision Recall Curve value, and Time is taken to build a model (In Seconds).

Table 2: Various Measurement of Classifiers on Dataset by Auto Color Correlogram Filter

\begin{tabular}{|c|c|c|c|c|c|c|c|c|c|c|c|c|c|c|c|}
\hline $\begin{array}{l}\text { S. } \\
\text { N } \\
\text { o }\end{array}$ & Category & $\begin{array}{c}\text { Classifi } \\
\text { er }\end{array}$ & $\begin{array}{c}\text { Time } \\
\text { Taken } \\
\text { to build } \\
\text { model }\end{array}$ & $\begin{array}{l}\text { Accu } \\
\text { racy }\end{array}$ & $\begin{array}{c}\text { Kap } \\
\text { pa } \\
\text { Stat } \\
\text { istic }\end{array}$ & $\begin{array}{c}\text { Mea } \\
\text { n } \\
\text { Abso } \\
\text { lute } \\
\text { Erro } \\
\text { r }\end{array}$ & $\begin{array}{c}\text { Roo } \\
\mathbf{t} \\
\text { Mea } \\
\mathbf{n} \\
\text { Squ } \\
\text { ared } \\
\text { Err } \\
\text { or }\end{array}$ & $\begin{array}{c}\text { Rela } \\
\text { tive } \\
\text { Abso } \\
\text { lute } \\
\text { Erro } \\
\text { r }\end{array}$ & $\begin{array}{c}\text { Roo } \\
t \\
\text { Rela } \\
\text { tive } \\
\text { Squ } \\
\text { ared } \\
\text { Err } \\
\text { or }\end{array}$ & $\begin{array}{c}\text { Preci } \\
\text { sion }\end{array}$ & $\begin{array}{c}\text { Re } \\
\text { cal } \\
1\end{array}$ & $\begin{array}{l}\text { F- } \\
\text { Meas } \\
\text { ure }\end{array}$ & $\begin{array}{l}\text { M } \\
\text { C } \\
\text { C }\end{array}$ & $\begin{array}{l}\mathbf{R} \\
\mathbf{O} \\
\mathbf{C}\end{array}$ & $\begin{array}{l}\mathbf{P} \\
\mathbf{R} \\
\mathbf{C}\end{array}$ \\
\hline 1 & Bayes & $\begin{array}{l}\text { BayesN } \\
\text { et }\end{array}$ & 1.80 & $\begin{array}{c}73.88 \\
\% \\
\end{array}$ & 0.60 & 0.13 & 0.32 & $\begin{array}{c}40.6 \\
6 \% \\
\end{array}$ & $\begin{array}{c}80.3 \\
0 \% \\
\end{array}$ & 0.75 & $\begin{array}{c}0.7 \\
4\end{array}$ & 0.74 & $\begin{array}{l}0 . \\
63\end{array}$ & $\begin{array}{l}0 . \\
92\end{array}$ & $\begin{array}{c}0 . \\
80\end{array}$ \\
\hline 2 & Functions & $\begin{array}{l}\text { Sequent } \\
\text { ial } \\
\text { Minima } \\
1 \\
\text { Optimi } \\
\text { zation } \\
\end{array}$ & 3.19 & $\begin{array}{c}72.65 \\
\%\end{array}$ & 0.56 & 0.28 & 0.36 & $\begin{array}{c}86.9 \\
8 \%\end{array}$ & $\begin{array}{c}89.7 \\
3 \%\end{array}$ & 0.72 & $\begin{array}{c}0.7 \\
3\end{array}$ & 0.70 & $\begin{array}{c}0 . \\
59\end{array}$ & $\begin{array}{l}0 . \\
82\end{array}$ & $\begin{array}{l}0 . \\
62\end{array}$ \\
\hline 3 & Lazy & IBK & 0.00 & $\begin{array}{c}75.06 \\
\%\end{array}$ & 0.61 & 0.14 & 0.30 & $\begin{array}{c}45.0 \\
5 \%\end{array}$ & $\begin{array}{c}72.9 \\
6 \%\end{array}$ & 0.74 & $\begin{array}{c}0.7 \\
5\end{array}$ & 0.74 & $\begin{array}{l}0 . \\
64\end{array}$ & $\begin{array}{l}0 . \\
90\end{array}$ & $\begin{array}{l}0 . \\
78\end{array}$ \\
\hline 4 & Ensemble & $\begin{array}{l}\text { Baggin } \\
\mathrm{g}\end{array}$ & 16.95 & $\begin{array}{c}79.97 \\
\% \\
\end{array}$ & 0.69 & 0.15 & 0.27 & $\begin{array}{c}44.5 \\
1 \% \\
\end{array}$ & $\begin{array}{c}66.1 \\
8 \% \\
\end{array}$ & 0.80 & $\begin{array}{c}0.8 \\
0 \\
\end{array}$ & 0.80 & $\begin{array}{l}0 . \\
71 \\
\end{array}$ & $\begin{array}{l}0 . \\
94 \\
\end{array}$ & $\begin{array}{r}0 . \\
85 \\
\end{array}$ \\
\hline 5 & Rules & JRip & 26.17 & $\begin{array}{c}75.77 \\
\% \\
\end{array}$ & 0.62 & 0.17 & 0.31 & $\begin{array}{c}52.2 \\
5 \% \\
\end{array}$ & $\begin{array}{c}76.3 \\
4 \% \\
\end{array}$ & 0.75 & $\begin{array}{c}0.7 \\
6 \\
\end{array}$ & 0.75 & $\begin{array}{l}0 . \\
64\end{array}$ & $\begin{array}{l}0 . \\
87\end{array}$ & $\begin{array}{l}0 . \\
74 \\
\end{array}$ \\
\hline 6 & Trees & $\begin{array}{l}\text { Rando } \\
\mathrm{m} \\
\text { Forest }\end{array}$ & 15.97 & $\begin{array}{c}80.96 \\
\%\end{array}$ & 0.70 & 0.14 & 0.20 & $\begin{array}{c}43.7 \\
3 \%\end{array}$ & $\begin{array}{c}64.4 \\
0 \%\end{array}$ & 0.81 & $\begin{array}{c}0.8 \\
1\end{array}$ & 0.81 & $\begin{array}{l}0 . \\
72\end{array}$ & $\begin{array}{l}0 . \\
95\end{array}$ & $\begin{array}{c}0 . \\
87\end{array}$ \\
\hline
\end{tabular}


The above table clearly shows that the BayesNet classifier is having $73.88 \%$ of accuracy level, The SMO classifier is having $72.65 \%$ of accuracy level, The Instance Based Classifier is having $75.06 \%$ of accuracy level, The Bagging classifier is having $79.97 \%$ of accuracy level, The JRip classifier is having $75.77 \%$ of accuracy level and Random Forest classifier is having $80.96 \%$ of accuracy level.

The BayesNet classifier is producing 0.60 of Kappa statistic value, The Sequential Minimal Optimization classifier is producing 0.56 of Kappa statistic value, The Instance Based Classifier is producing 0.61 of Kappa statistic value, The Bagging classifier is producing 0.69 of Kappa statistic value, The JRip classifier is producing 0.62 of Kappa statistic value and Random Forest classifier is producing 0.70 of Kappa statistic value.

The BayesNet classifier is having 0.13 of Mean Absolute Error value, The SMO classifier is having 0.28 of Mean Absolute Error value, The Instance Based Classifier is having 0.14 of Mean Absolute Error value, The Bagging classifier is having 0.15 of Mean Absolute Error value, The JRip classifier is having 0.17 of Mean Absolute Error value and Random Forest classifier is having 0.14 of Mean Absolute Error value.

The BayesNet model of Bayes category is producing 0.32 of RMSE value, The Sequential Minimal Classifier model of function category is producing 0.36 of RMSE value, The Instance Based model of Lazy category is producing 0.30 of RMSE value, The Bagging model of ensemble category is producing 0.27 of RMSE value, The JRip model of Rules category is producing 0.31 of RMSE value and Random Forest model of tree category is producing 0.20 of RMSE value.

The BayesNet classifier is having $40.66 \%$ of Relative Squared Error value, The SMO classifier is having $86.98 \%$ of Relative Squared Error value, The Instance Based Classifier is having $45.05 \%$ of Relative Squared Error value, The Bagging classifier is having $44.51 \%$ of Relative Squared Error value, The JRip classifier is having $52.25 \%$ of Relative Squared Error value and Random Forest classifier is having $43.73 \%$ of Relative Squared Error value.

The BayesNet model of Bayes category is producing $80.30 \%$ of Root Relative Squared Error (RRSE) value, The Sequential Minimal Classifier model of function category is producing $89.73 \%$ of RRSE value, The Instance Based model of Lazy category is producing $72.96 \%$ RRSE value, The Bagging model of ensemble category is producing $66.18 \%$ of RRSE value, The JRip model of Rules category is producing $76.34 \%$ of RRSE value and Random Forest model of tree category is producing $64.40 \%$ of RRSE value.

The BayesNet classifier is having 0.75 of Precision value, The SMO classifier is having 0.72 of Precision value, The Instance Based Classifier is having 0.74 of Precision value, The Bagging classifier is having 0.80 of Precision value, The JRip classifier is having 0.75 of Precision value and Random Forest classifier is having 0.81 of Precision value.

The BayesNet classifier is having 0.74 of Recall value, The SMO classifier is having 0.73 of Recall value, The Instance Based Classifier is having 0.75 of Recall value, The Bagging classifier is having 0.80 of Recall value, The JRip classifier is having 0.76 of Recall value and Random Forest classifier is having 0.81 of Recall value.

The BayesNet classifier is having 0.74 of F-Measure value, The SMO classifier is having 0.70 of FMeasure value, The Instance Based Classifier is having 0.74 of F-Measure value, The Bagging classifier is having 0.80 of F-Measure value, The JRip classifier is having 0.75 of F-Measure value and Random Forest classifier is having 0.81 of F-Measure value.

The BayesNet classifier is having 0.63 of Mathew Correlation Coefficient value, The SMO classifier is having 0.59 of Mathew Correlation Coefficient value, The Instance Based Classifier is having 0.64 of Mathew Correlation Coefficient value, The Bagging classifier is having 0.71 of Mathew Correlation Coefficient value, The JRip classifier is having 0.64 of Mathew Correlation Coefficient value and Random Forest classifier is having 0.72 of Mathew Correlation Coefficient value.

The BayesNet model of Bayes category is producing 0.92 of receiver operating characteristic curve (ROC) value, The Sequential Minimal Classifier model of function category is producing 0.82 of ROC value, The Instance Based model of Lazy category is producing 0.90 of ROC value, The Bagging model of ensemble category is producing 0.94 of ROC value, The JRip model of Rules category is producing 0.87 of ROC value and the Random Forest model of tree category is producing 0.95 of ROC value.

The BayesNet classifier is having 0.80 of precision recall curve value, The SMO classifier is having 0.62 of precision recall curve value, The Instance Based Classifier is having 0.78 of precision recall curve value, The Bagging classifier is having 0.85 of precision recall curve value, The JRip classifier is having 0.74 of precision recall curve value and Random Forest classifier is having 0.87 of precision recall curve value. 
The BayesNet classifier is taking 1.8 seconds to build a model, The SMO classifier is taking 3.19 seconds to build a model, The Instance Based Classifier is taking zero second to build a model, The Bagging classifier is taking 16.95 seconds to build a model, The JRip classifier is taking 26.17 seconds to build a model and Random Forest classifier is taking 15.97 seconds to build a model.

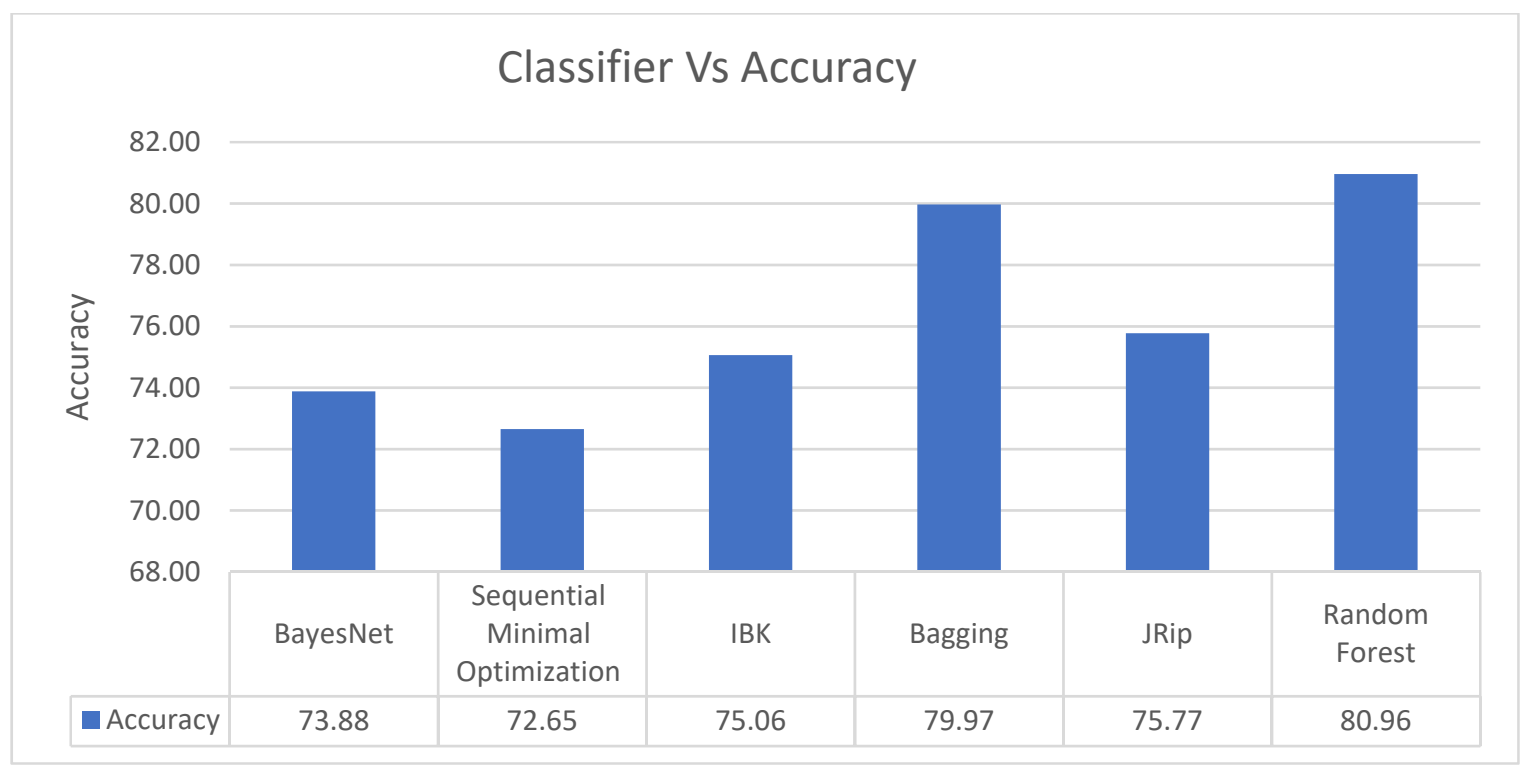

Figure 6: Graphical representations of various classifiers with their accuracy levels

The accuracies obtain from the selected classifiers are shown in Figure 6. This graph represents that the comparisons of accuracies for all the categories of the classifiers.

All classifiers are producing above $72 \%$ of accuracy level. The lowest accuracy is $72.65 \%$ which is produced by SMO model (SVM). The highest accuracy level is $80.96 \%$ which is produced by Random Forest classifier. Rest of the classifiers are having around $73.88 \%$ accuracy level to $79.77 \%$ of accuracy level.

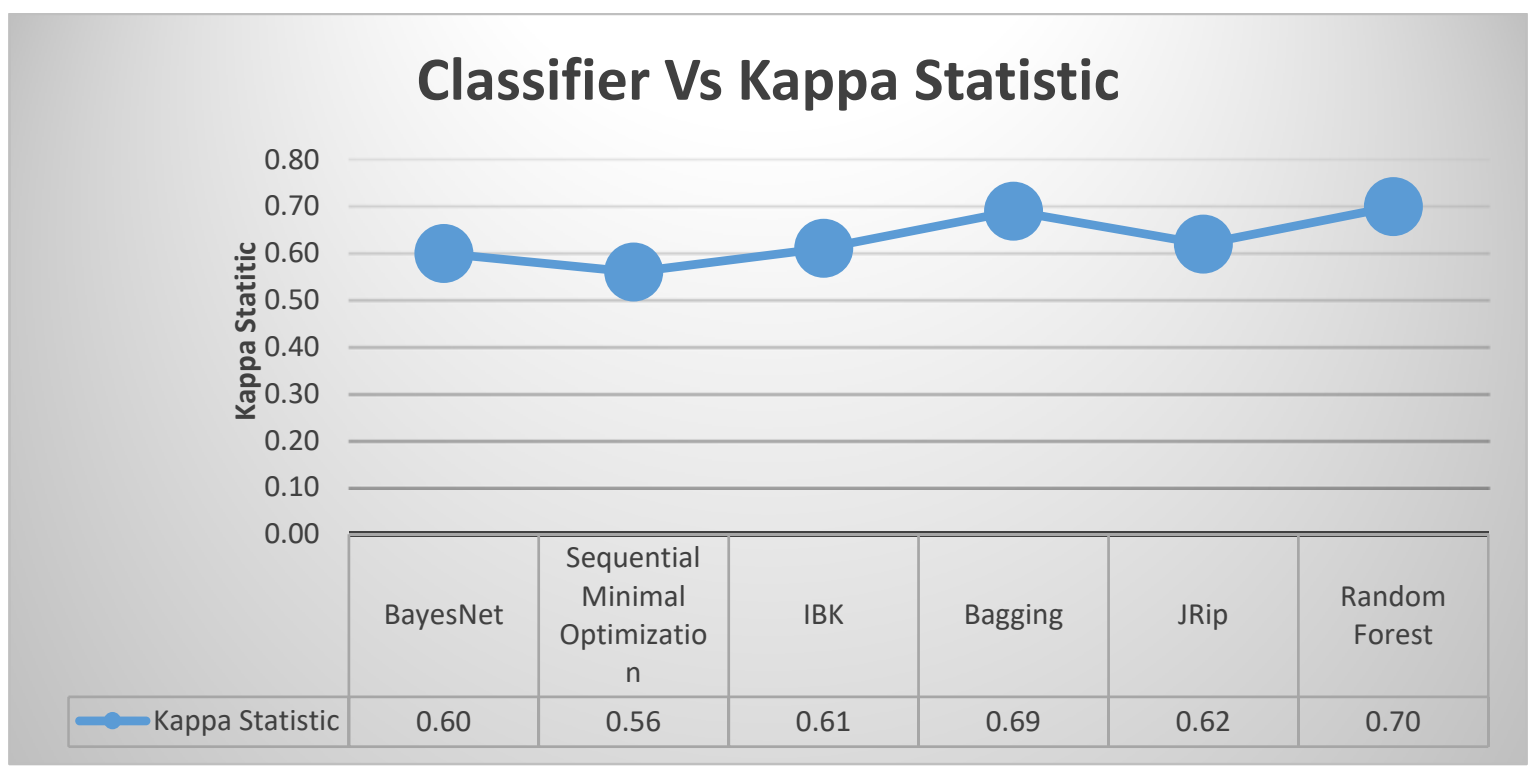

Figure 7: Graphical representations of various classifiers with their Kappa Statistic values

The kappa statistic values obtain from the selected classifiers are shown in Figure 7.This graph represents that the comparisons of kappa statistic for all the categories of the classifiers. 
The Sequential Minimal Optimization classifier is having moderate inter rater reliability of 0.56 of kappa statistic value. The remaining models like BayesNet of Bayes category, IBK of Lazy category, Bagging of Ensemble category, JRip of Rules category and Random Forest of Tree category models are having substantial inter rater reliability which is 0.60 kappa statistic value to 0.70 kappa statistic value.

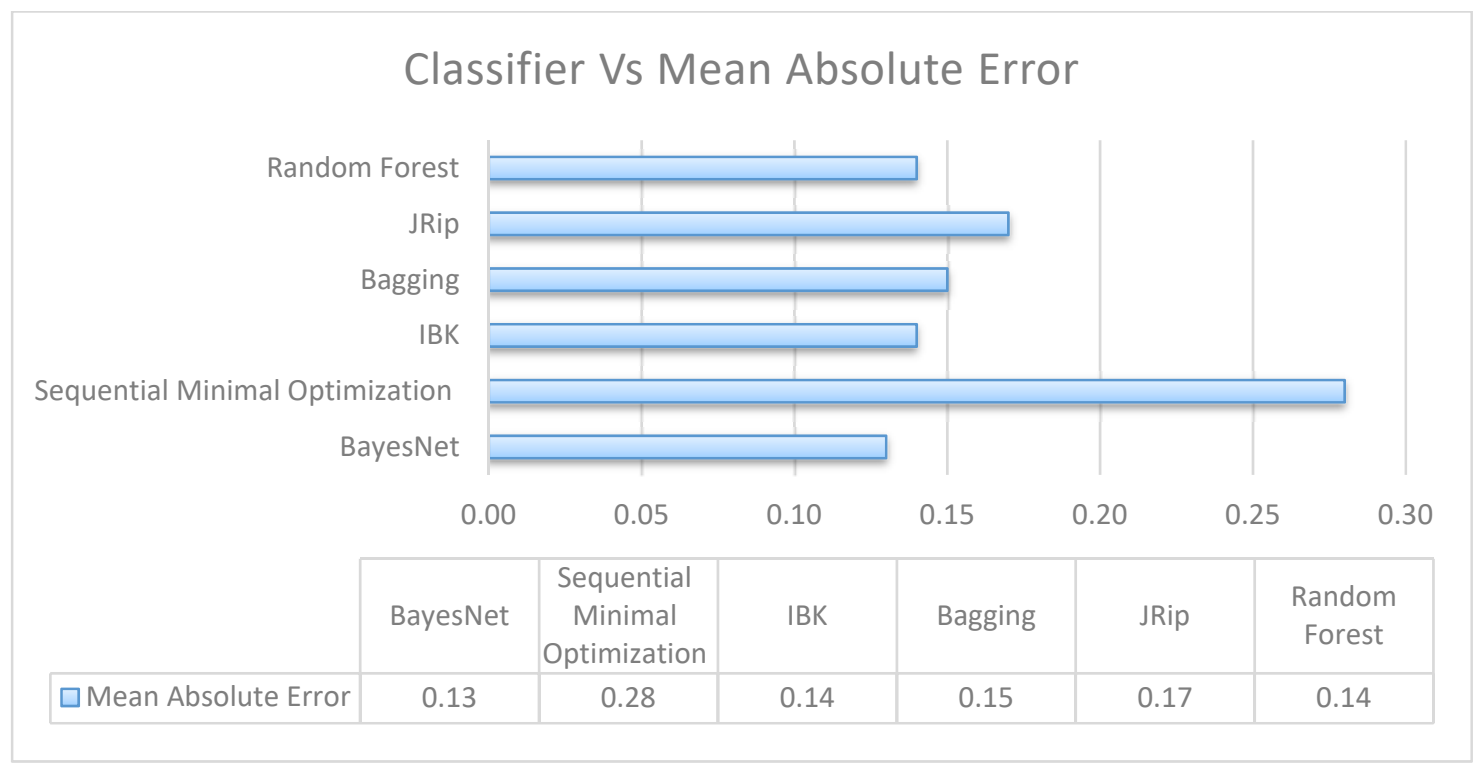

Figure 8: Graphical representations of ensemble classifiers with their Mean Absolute Error values

The mean absolute error values obtain from the selected classifiers are shown in Figure 8.This graph represents that the comparison of mean absolute error values of all the categories of the classifiers.

The Bayes classifier is having lowest Mean Absolute Error value which is 0.13 of mean absolute error value. The Sequential Minimal Optimization classifier is having highest Mean absolute Error value which is 0.28 of mean absolute error value. The Instance based classifier and Random Forest Classifier are having more or less similar Mean absolute Error value with BayesNet classifier which is 0.14 of mean absolute error value.

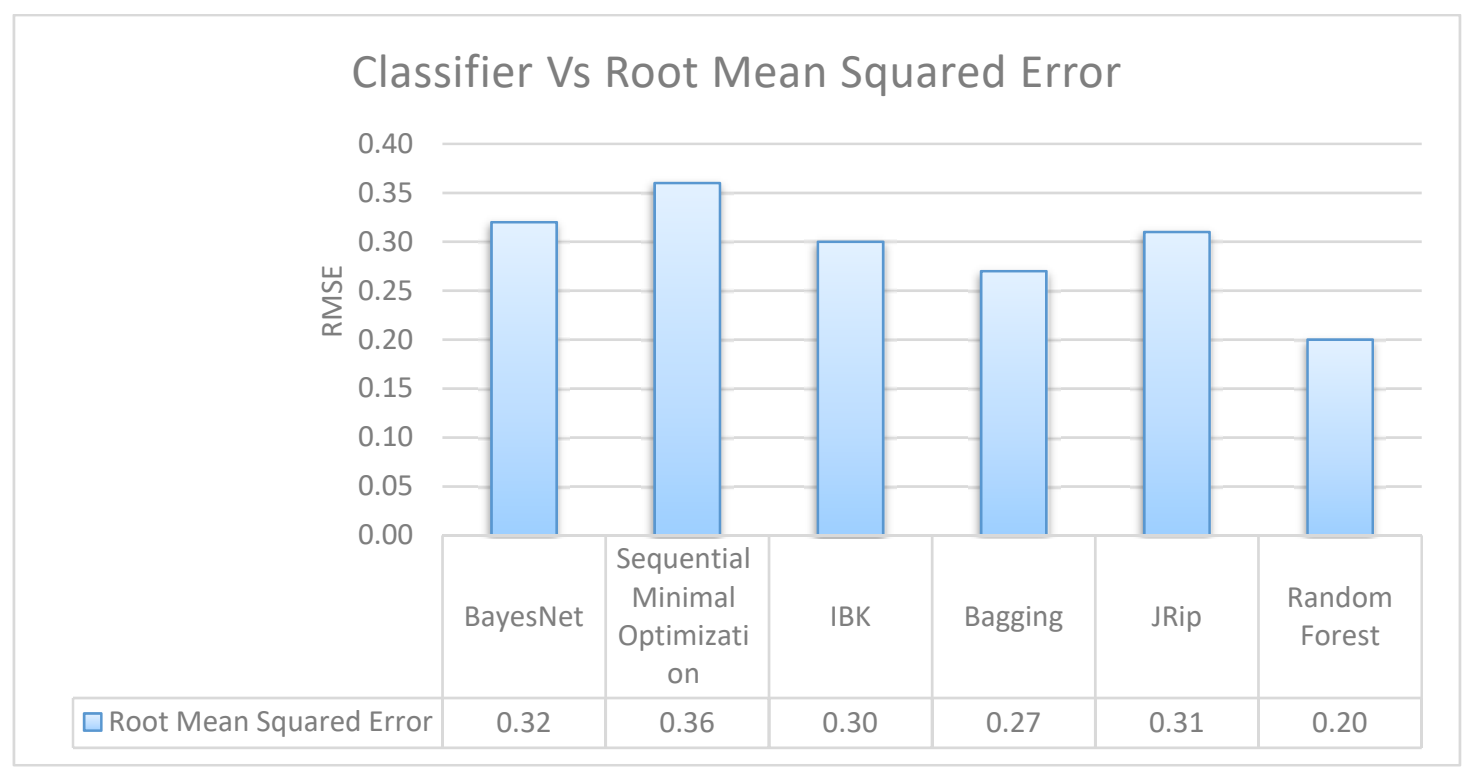

Figure 9: Graphical representations of various classifiers with their Root Mean Squared Error values

The root mean squared error (RMSE) values obtain from the selected classifiers are shown in Figure 9.This graph represents that the comparison of root mean squared error values of all the categories of the classifiers. 
The Random Forest Classifier is having lowest RMSE value which is 0.20 . The Highest RMSE value is 0.36 which is given by SMO model. Only the Bagging model of Meta category and Random Forest model of Tree category model are having less than 0.30 of RMSE value. Others are having above 0.30 of RMSE value like, IBk model is 0.30 of RMSE value, and JRip model is 0.31 of RMSE value.

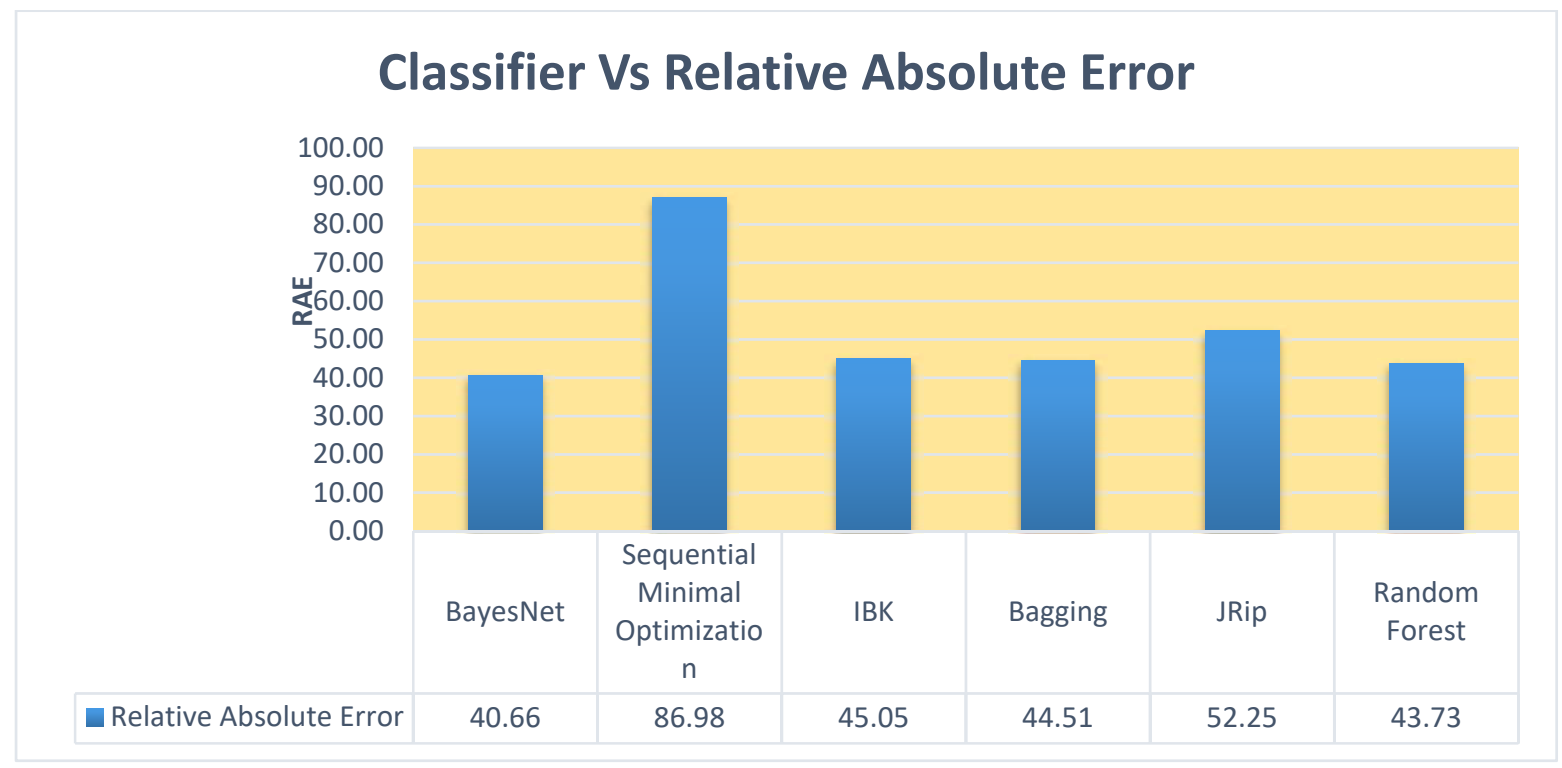

Figure 10 Graphical representations of various classifiers with their Relative Absolute Error values

The above figure shows that the relative absolute error (RAE) values obtain from the selected models. The lowest RAE value is $40.66 \%$ which is given by BayesNet model of Bayes category. The Highest RAE value is $86.98 \%$ which is given by Sequential Minimal Optimization model of function category. The others values are $43.73 \%$ of RAE value which is made by Random Forest model of tree category, $44.51 \%$ of RAE value which is given by Bagging model of ensemble category, $45.05 \%$ of RAE value which is produced by Instance Based model of Lazy category, and $52.25 \%$ of RAE value which is given by JRip model of Rules category.

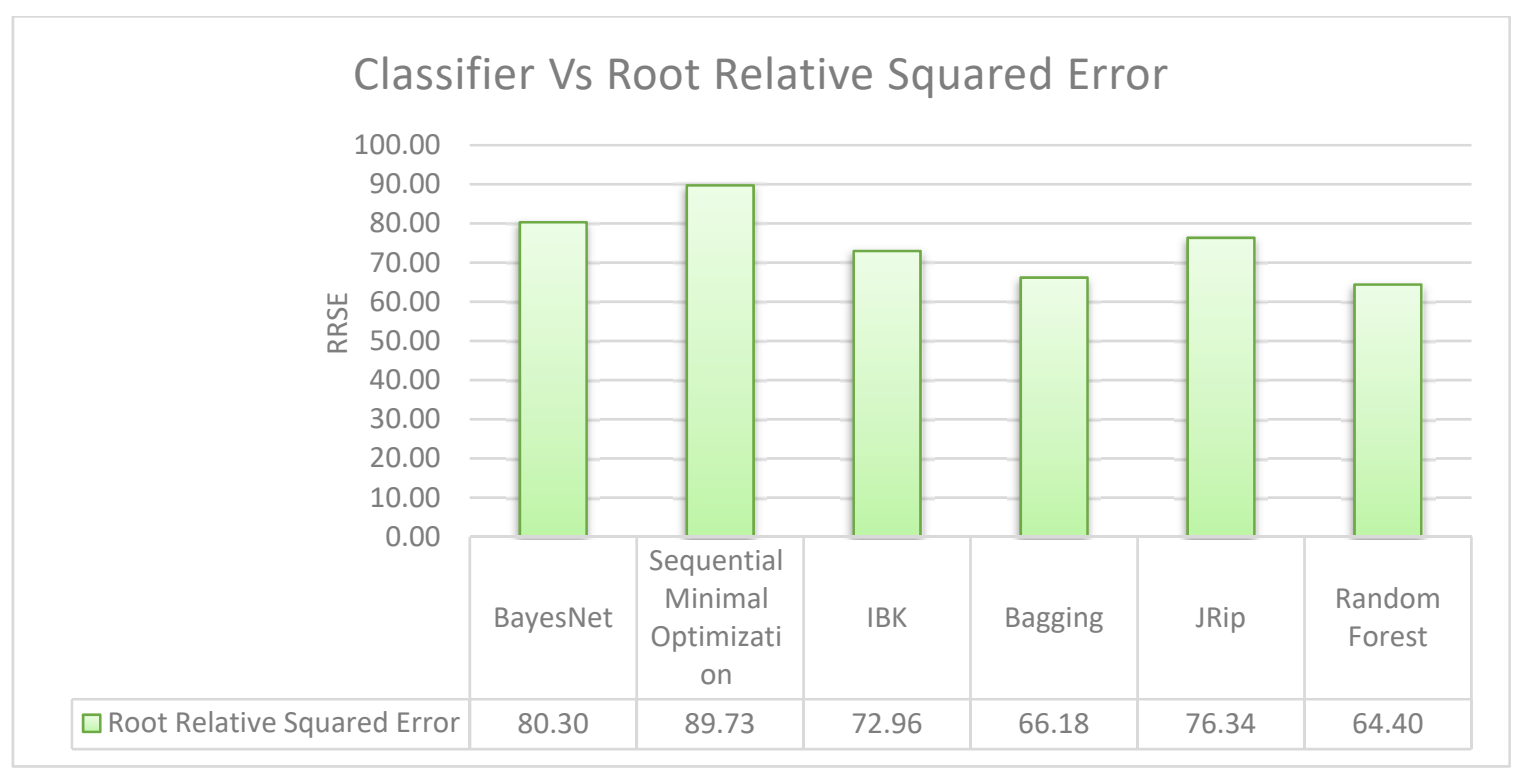

Figure 11: Graphical representations of various classifiers with their Root Relative Squared Error values models

The above diagram shows that the root relative squared error (RRSE) values obtain from the selected

The highest RRSE value is $89.73 \%$ which is given by SMO model of function category. The lowest RRSE value is $64.40 \%$ which is generated by Random Forest model of Trees category. 


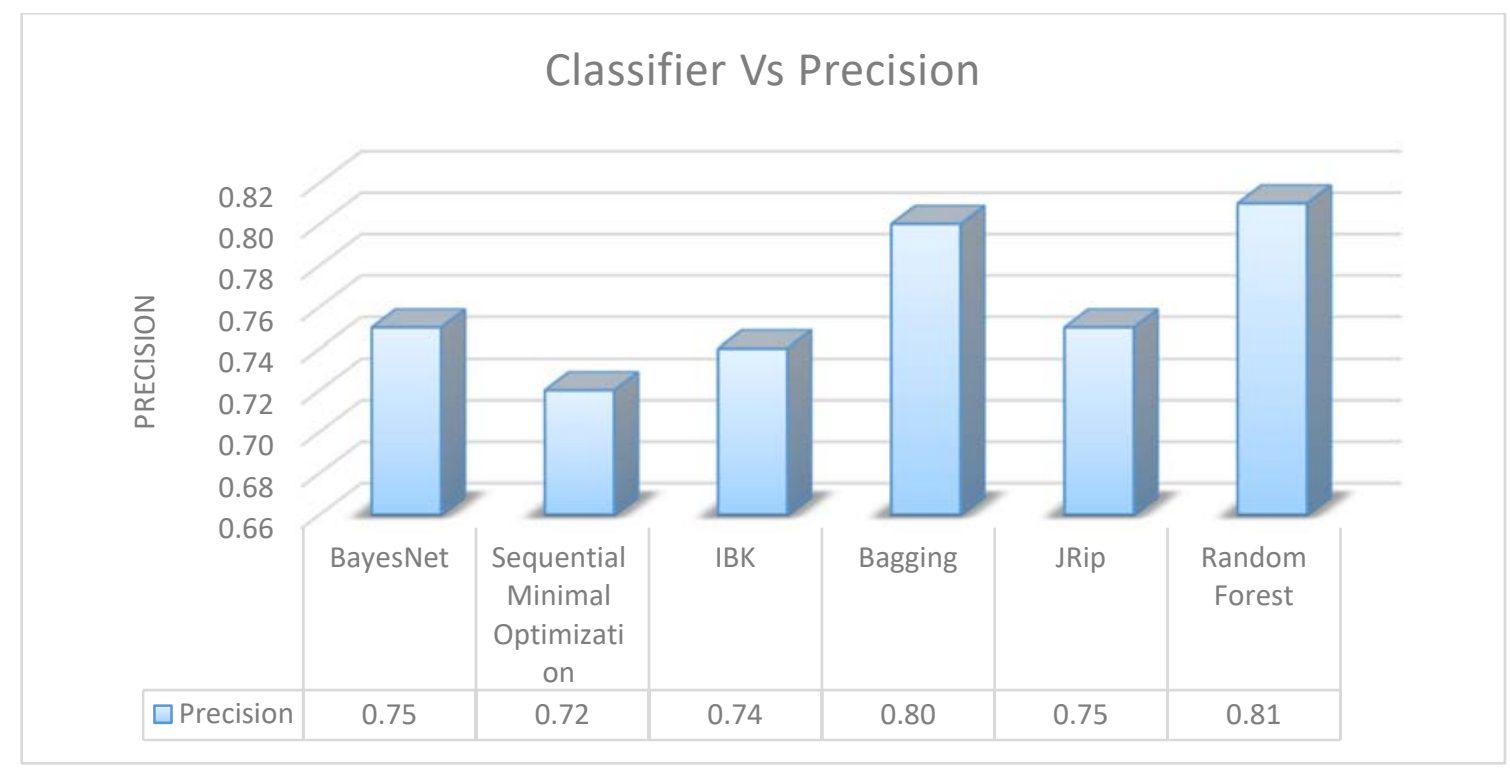

Figure 12: Graphical representations of various classifiers with their precision values

The above diagram shows that the precision values obtain from the selected models. The lowest precision value is given by SMO model of function category which is 0.72 of precision value and the maximum precision value is 0.81 by Random Forest model of Trees category.

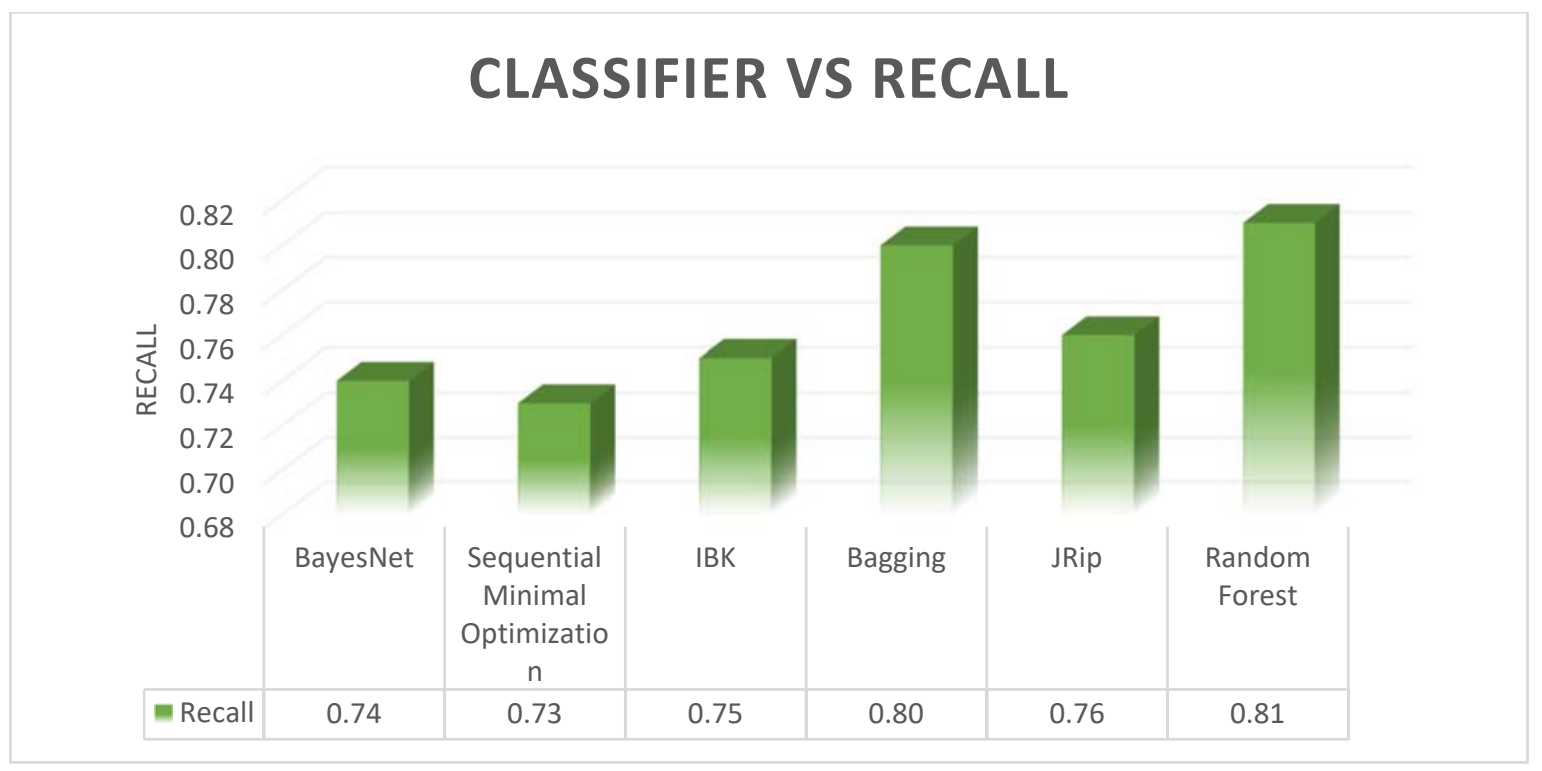

Figure 13: Graphical representations of various classifiers with their recall values

The recall values obtain from the selected classifiers are shown in Figure 12. This chart represents that the comparison of recall values of all the categories of the classifiers.

The maximum value of recall is 0.81 which produced by Random Forest algorithm and minimum value of recall is 0.73 which is produced by SMO model. 


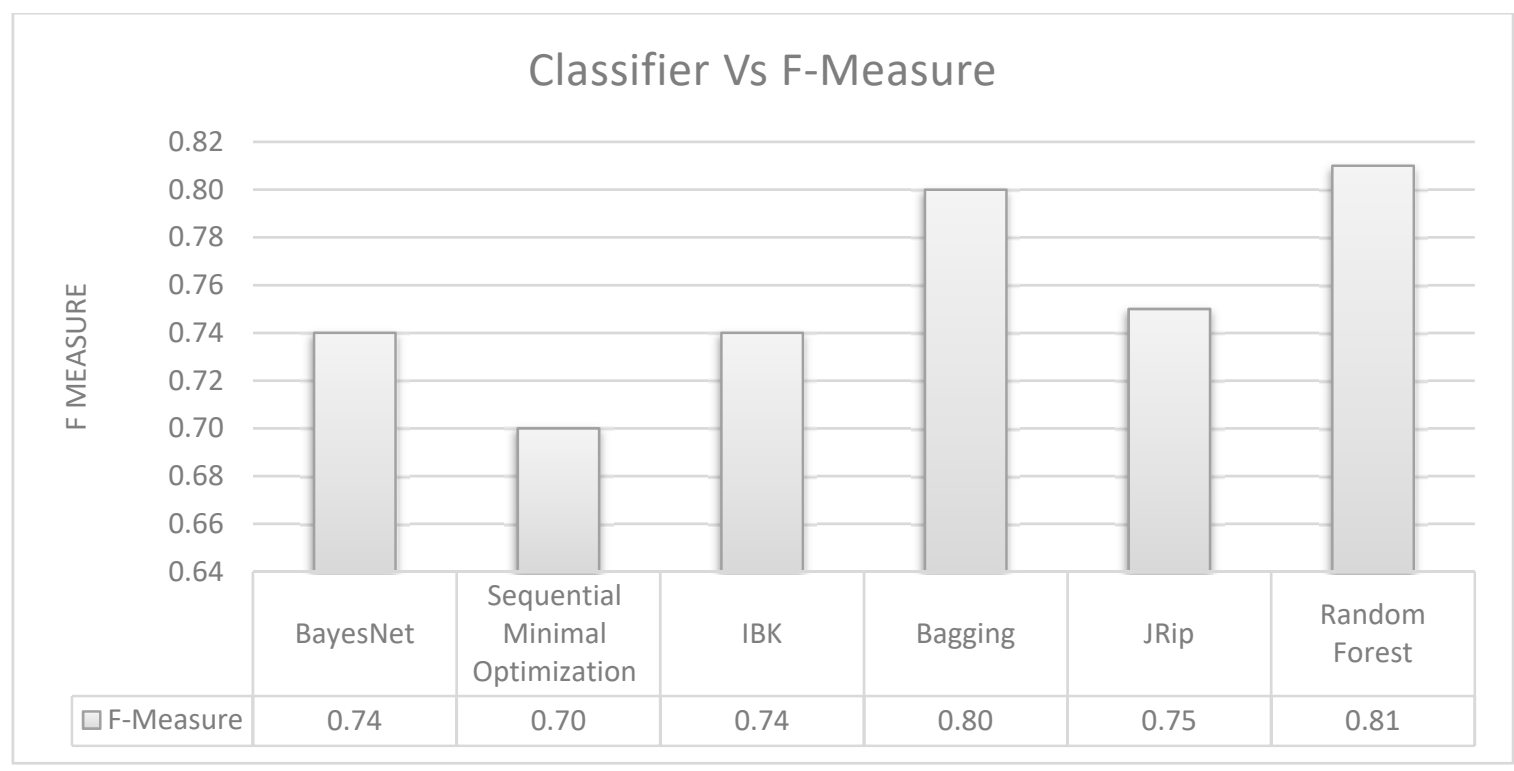

Figure 14: Graphical representations of various classifiers with their F- Measure values

The F- Measure values obtain from the selected classifiers are shown in Figure 13. This chart represents that the comparison of $\mathrm{F}$ - Measure values of all the categories of the classifiers.

The maximum F Measure value is 0.81 which produced by Random Forest algorithm and minimum F Measure value is 0.70 which is produced by SMO model.

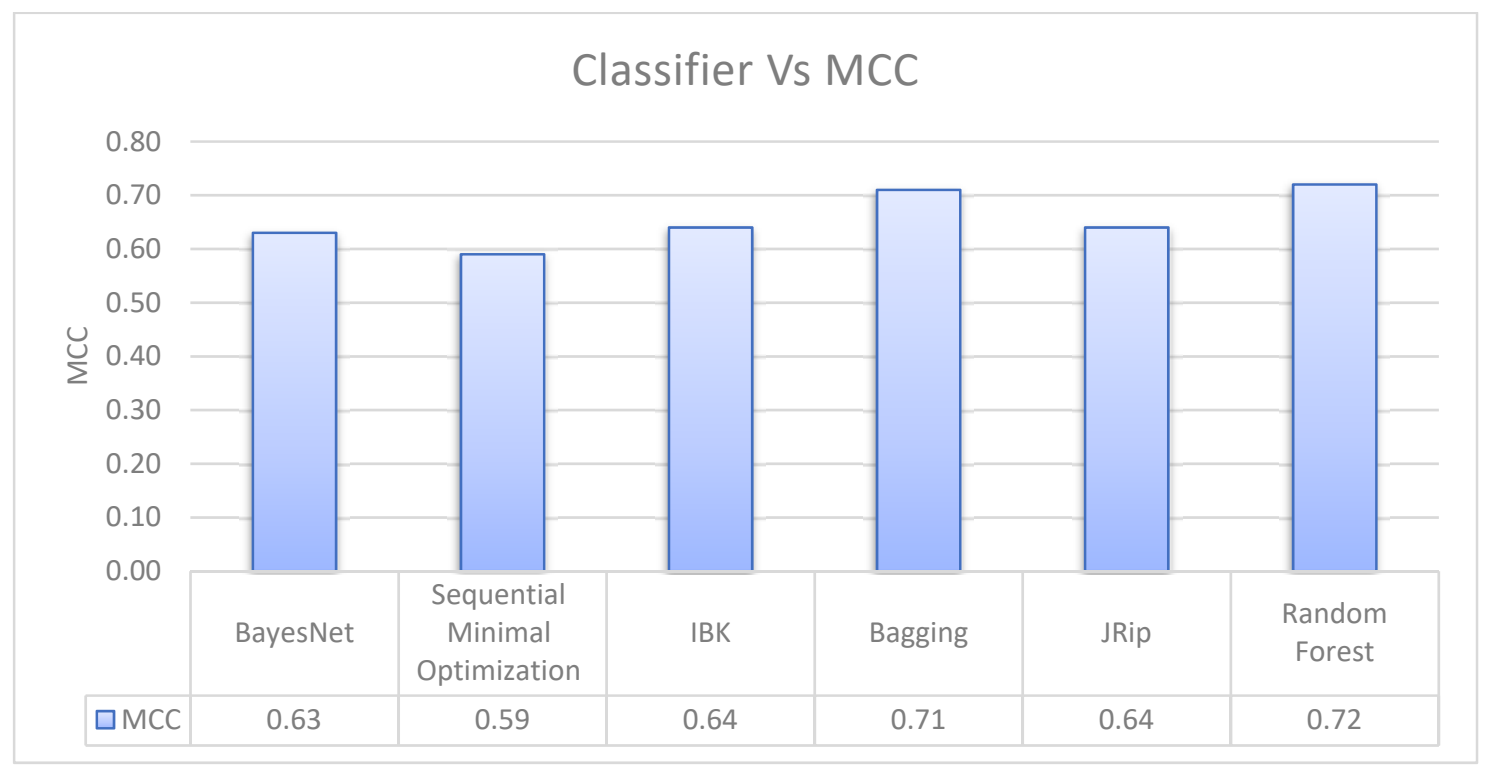

Figure 15: Graphical representations of various classifiers with their MCC values

The MCC values obtain from the selected classifiers are shown in Figure 14. This graph represents that the comparison of MCC values of all the categories of the classifiers.

The highest Mathew Correlation Coefficient value is 0.72 which produced by Random Forest algorithm and lowest Mathew Correlation Coefficient value is 0.59 which is produced by SMO model. 


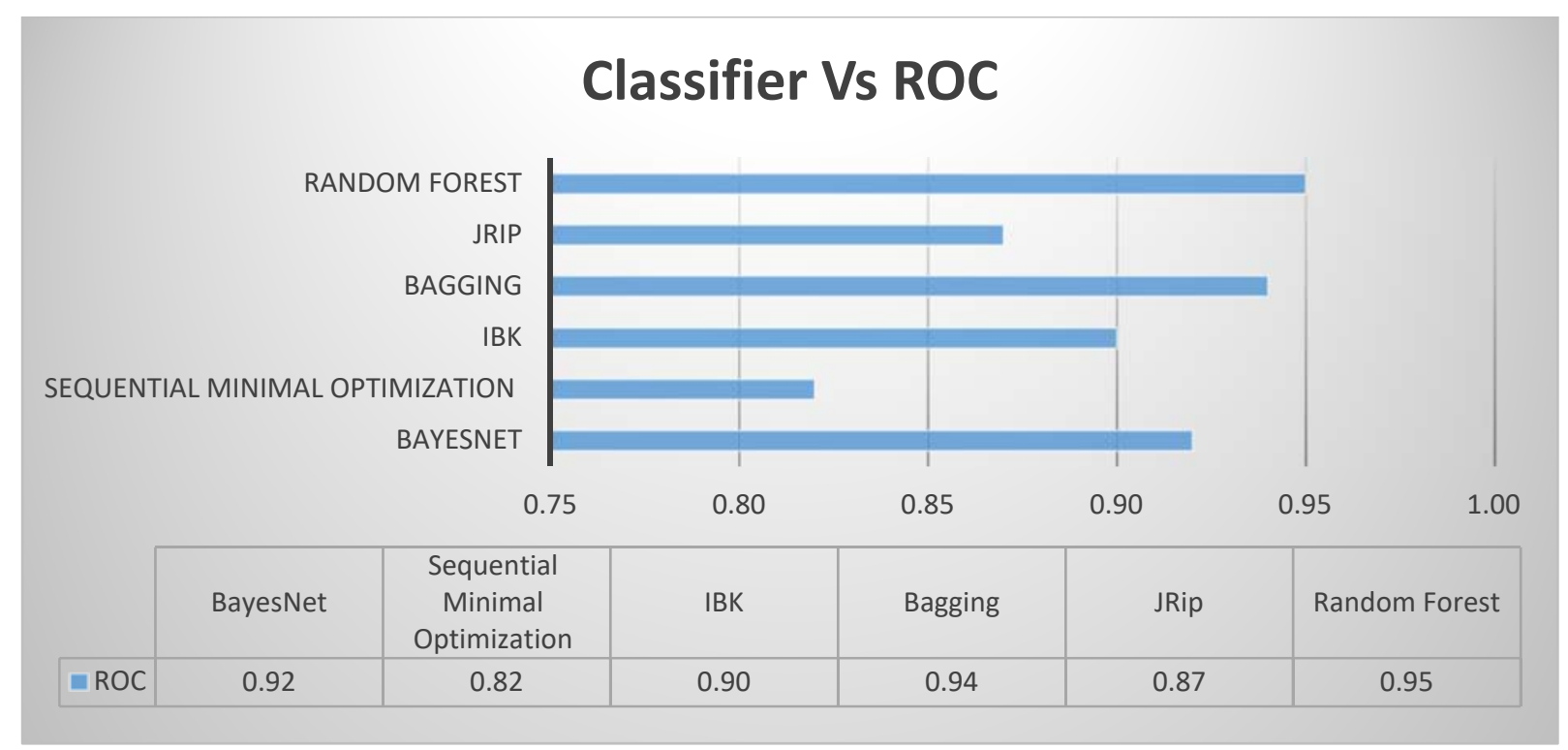

Figure 16: Graphical representations of various classifiers with their ROC values

The above diagram shows that the ROC values obtain from the selected models. The highest ROC value is 0.95 produced by Random Forest model and lowest ROC value is 0.82 produced by SMO model.

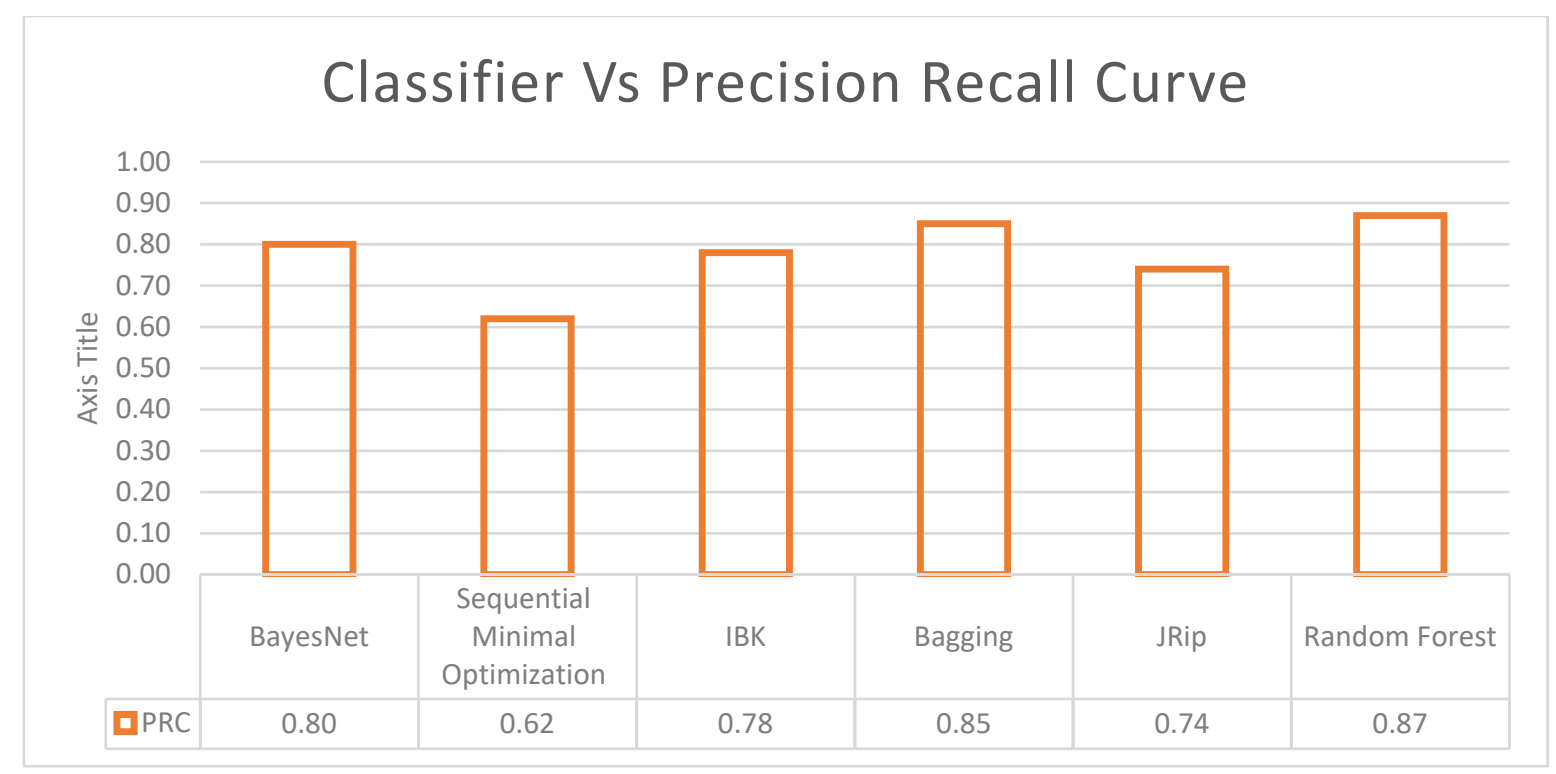

Figure 17: Graphical representations of various classifiers with their PRC values

The above diagram depicts that the PRC values obtain from the selected models. The highest PRC value is 0.87 which produced by Random Forest model and lowest PRC value is 0.62 which is produced by SMO model. 


\section{Classifier Vs Time Taken to build model(In Seconds)}

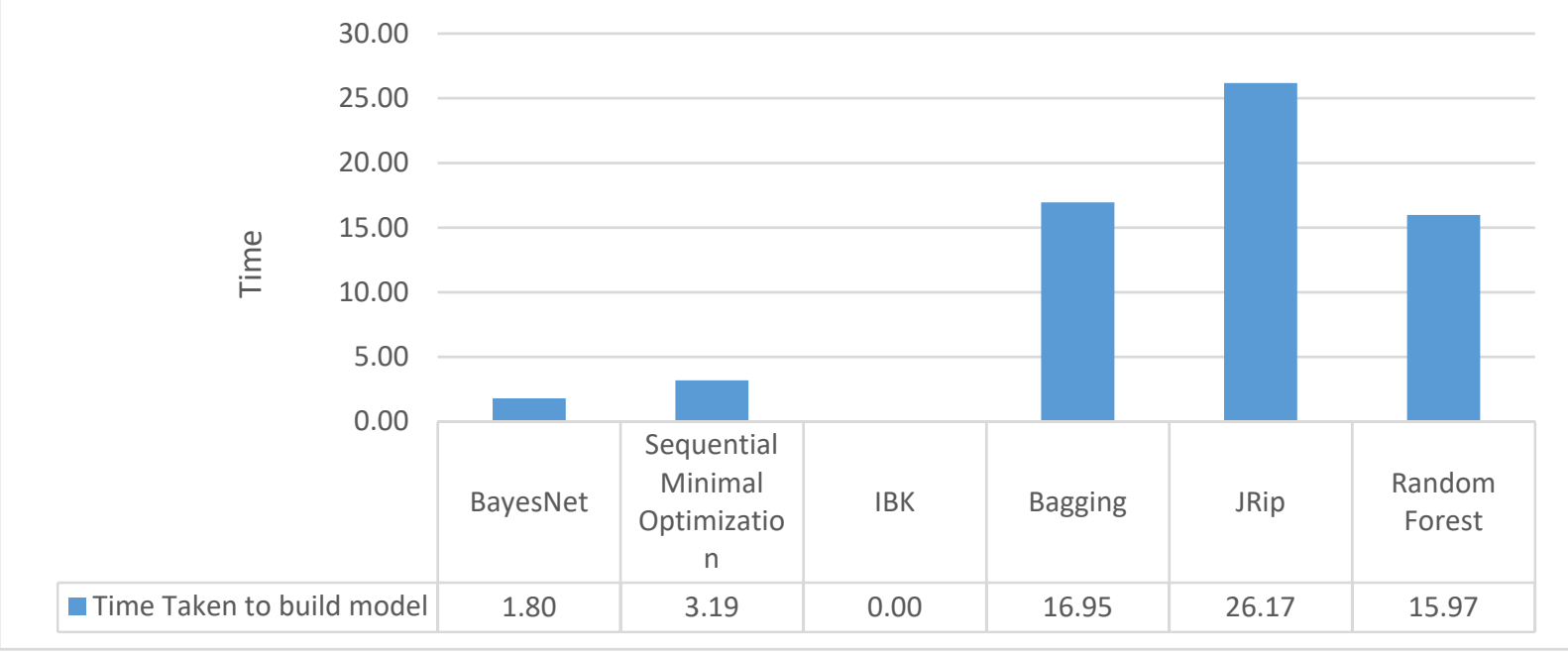

Figure 18: Graphical representations of various Classifiers with their time is taken to build a model

The above diagram shows that the time consumption to build each models obtain from the selected model

The Instance Based Classifier is taking zero second to build a model which is lowest time consumption of selected classifiers. The JRip Classifier is taking maximum time consumption to build a model which is 26.17 seconds. The Bagging and Random Forest classifier are taking to build the models respectively 16.95 seconds and 15.97 seconds. The BayeNet and SMO model are taking 1.80 seconds and 3.19 seconds to build a model.

\section{Conclusion}

This research work finds that the highest accuracy level is $80.96 \%$ which is given by Random Forest model of Trees category; the lowest accuracy is $72.65 \%$ which is given by SMO model of function category. The Random Forest model of Trees category is having substantial inter rater reliability which is 0.70 of kappa statistic value; The SMO model of function category is having moderate inter rater reliability of 0.56 of kappa statistic value. The BayesNet model is having lowest MAE value which is 0.13 and Random Forest Model are having more or less same MAE value which is 0.14 ; the SMO model is having highest MAE value which is 0.28 . The Random Forest Model is having lowest RMSE value which is 0.20 ; the Highest RMSE value is 0.36 which is given by SMO model; the lowest RAE value is $40.66 \%$ which is given by BayesNet model and are $43.73 \%$ of RAE value which is given by Random Forest model: The Highest RAE value is $86.98 \%$ which is given by SMO model. The highest RRSE value is $89.73 \%$ which is given by SMO model; the lowest RRSE value is $64.40 \%$ which is given by Random Forest model. The lowest precision value is given by SMO model which is 0.72 of precision value and the maximum precision value is 0.81 which is given by Random Forest model of Tree category. The maximum value of recall is 0.81 which given by Random Forest model of Tree category and minimum value of recall is 0.73 which is given by SMO model. The maximum F Measure value is 0.81 which given by Random Forest model and minimum F Measure value is 0.70 which is given by SMO model. The highest Mathew Correlation Coefficient value is 0.72 which given by Random Forest model of Trees category and lowest Mathew Correlation Coefficient value is 0.59 which is given by SMO model. The highest receiver operating characteristic curve value is 0.95 which given by Random Forest model and lowest receiver operating characteristic curve value is 0.82 which is given by SMO model. The highest precision recall curve value is 0.87 which given by Random Forest model of Trees category and lowest PRC value is 0.62 which is given by SMO model of function category. The Instance Based Model of Lazy category is taking zero second to build a model which is lowest time consumption of selected models. The JRip Model of Rules category is taking maximum time consumption to build a model which is 26.17 seconds. Finally this research work recommends that the Random Forest model of Trees category is most recommend model with Auto Color Correlogram Filter method in image classifications.

\section{References}

[1] M.E.H. Chowdhury, T. Rahman, A. Khandakar, R. Mazhar, M.A. Kadir, Z.B. Mahbub, K.R. Islam, M.S. Khan, A. Iqbal, N. Al-Emadi, M.B.I. Reaz, M. T. Islam, “Can AI help in screening Viral and COVID-19 pneumonia?” IEEE Access, Vol. 8, 2020, pp. 132665 132676.

[2] Rahman, T., Khandakar, A., Qiblawey, Y., Tahir, A., Kiranyaz, S., Kashem, S.B.A., Islam, M.T., Maadeed, S.A., Zughaier, S.M., Khan, M.S. and Chowdhury, M.E., 2020. Exploring the Effect of Image Enhancement Techniques on COVID-19 Detection using Chest X-ray Images. arXiv preprint arXiv:2012.02238.

[3] Ayyappan.G et al. SMO classification for cervical cancer dataset by applying various kernels, Indian Journal of Computer Science and Engineering (IJCSE), Volume No.10 Issue No.1 Feb-Mar 2019, Page No: 28-30, e-ISSN: 0976-5166, p-ISSN: 2231-3850. 
[4] Alshazly H, Linse C, Abdalla M, Barth E, Martinetz T. 2021. COVID-Nets: deep CNN architectures for detecting COVID-19 using chest CT scans. PeerJ Comput. Sci. 7:e655 http://doi.org/10.7717/peerj-cs.655

[5] https://bimcv.cipf.es/bimcv-projects/bimcv-covid19/\#1590858128006-9e640421-6711

[6] https://github.com/ml-workgroup/covid-19-image-repository/tree/master/png

[7] https://sirm.org/category/senza-categoria/covid-19/

[8] https://eurorad.org

[9] https://github.com/ieee8023/covid-chestxray-dataset

[10] https://figshare.com/articles/COVID_19_Chest_X-Ray_Image_Repository/12580328

[11] https://github.com/armiro/COVID-CXNet

[12] https://www.kaggle.com/c/rsna-pneumonia-detection-challenge/data

[13] https://www.kaggle.com/paultimothymooney/chest-xray-pneumonia

[14] S. A. Harmon, T. H. Sanford, S. Xu, E. B. Turkbey, H. Roth, Z. Xu, et al., "Artificial intelligence for the detection of COVID-19 pneumonia on chest CT using multinational datasets," Nature Communications, vol. 11 (1), p. 4080(2020/08/14 2020)

[15] COVID-19 CORONAVIRUS PANDEMIC [Online]. Available: https://www.worldometers.info/coronavirus/. [Accessed on 03-102021]

[16] Y. Yang, M. Yang, C. Shen, F. Wang, J. Yuan, J. Li, et al., "Evaluating the accuracy of different respiratory specimens in the laboratory diagnosis and monitoring the viral shedding of 2019-nCoV infections. medRxiv 2020.02. 11.20021493 [Preprint]. 17 February 2020," Preprint] doi: 0.1101/2020.02, vol. 11)

[17] Ayyappan.G et al. A Novel Classification Approach -1 on Breast Tissue dataset, Indian Journal of Computer Science and Engineering (IJCSE), Volume No.9 Issue No.4 Aug-Sep 2018, Page No: 115-118, e-ISSN: 0976-5166, p-ISSN: 2231-3850.

[18] M. Yu, D. Xu, L. Lan, M. Tu, R. Liao, S. Cai, et al., "Thin-section Chest CT Imaging of Coronavirus Disease 2019 Pneumonia: Comparison Between Patients with Mild and Severe Disease," Radiology: Cardiothoracic Imaging, vol. 2 (2), p. e200126(2020)

[19] Ayyappan.G et al. Heart Disease Data Set Classifications: Comparisons of Correlation Co Efficient by Applying Various Parameters in Gaussian Processes, Indian Journal of Computer Science and Engineering (IJCSE), Volume No.9 Issue No.5 Oct-Nov 2018, Page No: 130-134, e-ISSN: 0976-5166, p-ISSN: 2231-3850.

[20] T. Rahman, M. E. Chowdhury, A. Khandakar, K. R. Islam, K. F. Islam, Z. B. Mahbub, et al., "Transfer Learning with Deep Convolutional Neural Network (CNN) for Pneumonia Detection using Chest X-ray," Applied Sciences, vol. 10 (9), p. 3233(2020)

[21] T. Rahman, A. Khandakar, M. A. Kadir, K. R. Islam, K. F. Islam, R. Mazhar, et al., "Reliable Tuberculosis Detection using Chest Xray with Deep Learning, Segmentation and Visualization," IEEE Access, vol. 8 pp. 191586-191601(2020)

[22] A. Tahir, Y. Qiblawey, A. Khandakar, T. Rahman, U. Khurshid, F. Musharavati, et al., "Coronavirus: Comparing COVID-19, SARS and MERS in the eyes of AI," arXiv preprint arXiv:2005.11524, 2020)

[23] M. E. Chowdhury, T. Rahman, A. Khandakar, R. Mazhar, M. A. Kadir, Z. B. Mahbub, et al., "Can AI help in screening viral and COVID19 pneumonia?," IEEE Access, vol. 8 pp. 132665-132676(2020)

[24] T. Ai, Z. Yang, H. Hou, C. Zhan, C. Chen, W. Lv, et al., "Correlation of chest CT and RT-PCR testing in coronavirus disease 2019 (COVID-19) in China: a report of 1014 cases," Radiology, p. 200642(2020)

[25] Y. Fang, H. Zhang, J. Xie, M. Lin, L. Ying, P. Pang, et al., "Sensitivity of chest CT for COVID-19: comparison to RT-PCR," Radiology, p. 200432(2020)

[26] T. Rahman, A. Khandakar, M. A. Kadir, K. R. Islam, K. F. Islam, R. Mazhar, et al., "Reliable Tuberculosis Detection using Chest Xray with Deep Learning, Segmentation and Visualization," arXiv preprint arXiv:2007.14895, 2020)

[27] Ayyappan.G et al. Heart Disease Data Set Classifications: Comparisons of Correlation Co Efficient by Applying Various Functions, Indian Journal of Computer Science and Engineering (IJCSE), Volume No.9 Issue No.5 Oct-Nov 2018, Page No: 135-140, e-ISSN: 0976-5166, p-ISSN: 2231-3850.

\section{Authors Profile}
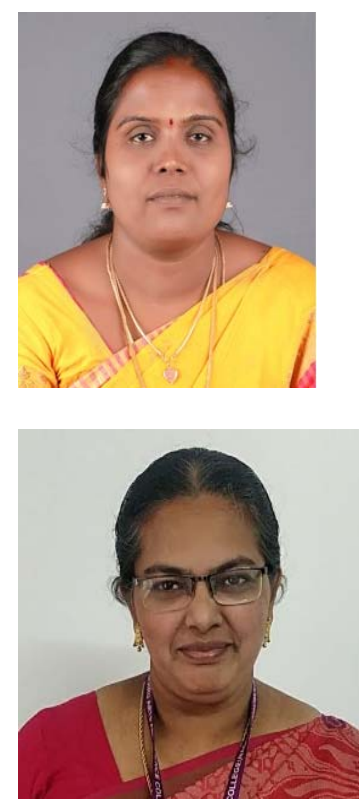

Mrs. S.M.Manimegalai, Ph. D Research scholar, Vivekanandha College of Arts and Science for Women (A), Elayampalayam-637205, Tiruchengode. Having 14 Years of teaching experience in DRBCCC Hindu College at Pattabiram-72. My area of interest is Image Processing and Machine Learning, and AI. Participated 20 FDP programmes and 15 seminars and workshops. I have published and presented 5 Research article in International conference. I received 2 times best paper award in International Conference, at Hindustan College of Arts and Science at Kelambakkam, chennai-603103.

Dr. T. Ramaprabha, I have been working as Professor in Department of Information Technology, Nehru Arts and Science College, Coimbatore. I am having 24 years of teaching and 18 years of research experience. I published more than 42 research articles in international journals and conferences. I completed 9 funded projects and committed with various academic bodies. Now, I am guiding $6 \mathrm{Ph} . \mathrm{D}$. scholars. My area of interest is Image processing, Data mining, Machine learning and Networking. 